\title{
Choosing wisely? Quantifying the extent of three low value psychotropic prescribing practices in Australia
}

\author{
Jonathan Brett ${ }^{{ }^{*}}$ (D), Helga Zoega ${ }^{1,2}$, Nicholas A. Buckley ${ }^{3}$, Benjamin J. Daniels ${ }^{1}$, Adam G. Elshaug $^{4}$ and
} Sallie-Anne Pearson ${ }^{1,4}$

\begin{abstract}
Background: The global Choosing Wisely campaign has identified the following psychotropic prescribing as low-value (harmful or wasteful): (1) benzodiazepine use in the elderly, (2) antipsychotic use in dementia and (3) prescribing two or more antipsychotics concurrently. We aimed to quantify the extent of these prescribing practices in the Australian population.

Methods: We applied indicators to dispensing claims of a 10\% random sample of Australian Pharmaceutical Benefits Scheme beneficiaries to quantify annual rates of each low-value practice from 2013 to 2016. We also assessed patient factors and direct medicine costs (extrapolated to the entire Australian population) associated with each practice in 2016.

Results: We observed little change in the rates of the three practices between 2013 and 2016. In 2016, 15.3\% of people aged $\geq 65$ years were prescribed a benzodiazepine, $0.5 \%$ were prescribed antipsychotics in the context of dementia and $0.2 \%$ of people aged $\geq 18$ years received two or more antipsychotics concurrently. The likelihood of elderly people receiving benzodiazepines or antipsychotics in the context of dementia increased with age and the likelihood of receiving all three practices increased with comorbidity burden. In 2016, direct medicine costs to the government of all three practices combined, extrapolated to national figures, were > \$21 million AUD.

Conclusions: Our indicators suggest that the frequency of these three practices has not changed appreciably in recent years and that they incur significant costs. Worryingly, people with the greatest risk of harm from these prescribing practices are often the most likely to receive them.
\end{abstract}

Keywords: Elderly, Benzodiazepines, Antipsychotics, Dementia, Polypharmacy, Choosing wisely

\section{Background}

The conception of the global Choosing Wisely campaign has heralded a renewed interest in addressing low-value care; medical practices such as test ordering, procedures or prescribing that are either harmful or wasteful [1]. The Choosing Wisely campaign is a physician-led, grass-root program which generates evidence-based 'top 5 lists' of practices considered low-value across a breadth of medical specialties $[2,3]$. Choosing Wisely was conceived in the U.S. in 2012 and has since spread globally across Europe

\footnotetext{
* Correspondence: J.Brett@unsw.edu.au

${ }^{1}$ Medicines Policy Research Unit, Centre for Big Data Research in Health,

University of New South Wales, Sydney, NSW 2052, Australia

Full list of author information is available at the end of the article
}

and Asia to over 20 countries around the world, launching in Australia in April 2015 [4]. The campaign nominates low-value practices to be focal points of conversations between patients and physicians with the ultimate aim of promoting alternative, more appropriate care. Developing indicators to quantify the extent of low-value medical practices allows policy priorities to be set and any changes following quality improvement strategies to be measured.

The majority of previous population-based research on low-value care has focused on medical tests and procedures [5], with limited attention to low-value prescribing practices [6]. This is despite the fact that when the nominated low-value practices in each country participating in the Choosing Wisely campaign are pooled, prescribing

(c) The Author(s). 2018 Open Access This article is distributed under the terms of the Creative Commons Attribution 4.0 International License (http://creativecommons.org/licenses/by/4.0/), which permits unrestricted use, distribution, and reproduction in any medium, provided you give appropriate credit to the original author(s) and the source, provide a link to the Creative Commons license, and indicate if changes were made. The Creative Commons Public Domain Dedication waiver (http://creativecommons.org/publicdomain/zero/1.0/) applies to the data made available in this article, unless otherwise stated. 
practices constitute nearly one quarter of all practices [6]. Of these, psychotropic prescribing practices feature heavily, and while measuring inappropriate psychotropic use in routinely collected data is not novel, examining them through the lens of low-value care is a relatively recent endeavor.

Most efforts to quantify low-value prescribing to date, such as the Australian Atlas of Variation in Health Care [7], have been based on aggregated dispensing claims data. Within the constraints of data content, individuallevel analyses enhance measurement approaches by allowing individuals receiving low-value prescribing practices to be more accurately identified.

Our overall objective was to develop indicators that can be applied to Pharmaceutical Benefits Scheme (PBS) data to quantify three low-value psychotropic prescribing practices in Australia. These practices were chosen as they were mentioned repeatedly within and between pooled national Choosing Wisely lists [6] and were conducive to measurement approaches using dispensing claims data. These practices are: (1) don't use benzodiazepines in the elderly, (2) avoid antipsychotics for dementia and (3) don't routinely prescribe two or more antipsychotics concurrently. Specifically, we applied indicators to estimate the annual rates of these practices between 2013 and 2016 and their association with patient age, sex and co-morbidity. While this may not capture changes resulting from the launch of Choosing Wisely in Australia, it provides an understanding of any underlying trends. We also estimated the direct costs of the medicines involved in each low-value psychotropic prescribing practice to the Australian government and patients.

\section{Methods}

\section{Study setting, data and population}

Australia has a publicly funded universal healthcare system entitling all citizens and permanent residents to subsidised access to prescription medicines via the Pharmaceutical Benefits Scheme (PBS). In this study, we used PBS dispensing records from 2010 to 2016 for a $10 \%$ random sample of all persons eligible to receive PBS-listed medicines. This is a standard dataset provided by the Australian Government Department of Human Services for analytical use and captures the majority of community based medicine dispensing [8]. Our study population consisted of all people 18 years and older within the PBS $10 \%$ sample with at least one dispensing record during the study period, 1 January 2013 through 31 December 2016.

\section{Medicines of interest}

We included medicines belonging to Anatomical Therapeutic Chemical Classification (ATC) classes antipsychotics (NO5A), benzodiazepines (NO5BA, NO5CD and NO3AE) and anti-dementia medicines (NO6D) [9] that were PBS-subsidised in Australia during the study period (see Additional file 1: Table S1 for full details of medicines and ATC codes).

\section{Indicators of low-value prescribing practices}

Since some measures require specific clinical information that is not contained within available dispensing claims data, we developed primary and secondary indicators for each low-value practice to illustrate the sensitivity of measurement to indicator definition (Table 1).

In this study, we defined annual incident (new) use as a dispensing of a medicine in a given year with no dispensings of that medicine in the 12 months prior and annual prevalent use as any dispensings of that medicine in a given year. We defined concurrent use of two or more antipsychotics (hereafter referred to as antipsychotic polypharmacy) as an overlap in antipsychotic exposure of > 60 days; an intentionally conservative measure that has been validated in dispensing claims data $[10,11]$. The duration of exposure following a

Table 1 Primary and secondary indicators applied to Pharmaceutical Benefits Scheme (PBS) dispensing claims data to measure each low-value prescribing practice

\begin{tabular}{|c|c|c|c|}
\hline \multirow[t]{2}{*}{ Low-value practice } & \multicolumn{3}{|l|}{ Indicator } \\
\hline & Primary & Secondary & Secondary indicator justification \\
\hline $\begin{array}{l}\text { Don't use benzodiazepines in } \\
\text { the elderly }\end{array}$ & $\begin{array}{l}\text { People } \geq 65 \text { years with prevalent } \\
\text { benzodiazepine use }\end{array}$ & $\begin{array}{l}\text { People } \geq 65 \text { years with incident } \\
\text { (new) benzodiazepine use }\end{array}$ & $\begin{array}{l}\text { New benzodiazepine use defines } \\
\text { a narrower population of recipients } \\
\text { of this practice than prevalent use }\end{array}$ \\
\hline Avoid antipsychotics for dementia & $\begin{array}{l}\text { People } \geq 65 \text { years dispensed an } \\
\text { anti-dementia medicine in the same } \\
\text { year or the three calendar years prior } \\
\text { to being dispensed an antipsychotic }{ }^{a}\end{array}$ & $\begin{array}{l}\text { People } \geq 65 \text { years with incident } \\
\text { (new) antipsychotic use }\end{array}$ & $\begin{array}{l}\text { Incident antipsychotic use is unlikely } \\
\text { to be for an indication where the } \\
\text { evidence of risk and benefit is clear } \\
\text { (schizophrenia or bipolar disorder) }\end{array}$ \\
\hline $\begin{array}{l}\text { Don't routinely prescribe two or } \\
\text { more antipsychotics concurrently }\end{array}$ & $\begin{array}{l}\text { People } \geq 18 \text { years dispensed two or } \\
\text { more antipsychotics with overlapping } \\
\text { treatment exposures of } 60 \text { days }\end{array}$ & $\begin{array}{l}\text { As for primary but excluding } \\
\text { long acting depot preparations }\end{array}$ & $\begin{array}{l}\text { Concomitant use of a long acting } \\
\text { depot with an oral antipsychotic may } \\
\text { be necessary during initial stages of } \\
\text { therapy while dosing is being optimized }\end{array}$ \\
\hline
\end{tabular}

${ }^{a}$ Anti-dementia medicines are PBS subsidised for Alzheimer's dementia but may be used in other forms of dementia in practice [34]

${ }^{\mathrm{b}}$ Long acting depot preparations are identified in Additional file 1: Table S1 
single antipsychotic dispensing was estimated as the time by which $75 \%$ of people had a repeat dispensing the same antipsychotic as previously described [10].

\section{Data analysis \\ Annual rates of low-value psychotropic prescribing (2013- 2016)}

We applied each indicator to estimate the number of persons experiencing the corresponding low-value practice in each calendar year. We divided this by the corresponding mid-calendar year population estimates from the Australian Bureau of Statistics (ABS) [12] for people in the relevant age group to calculate the annual rates of each prescribing practice.

We used different denominators to illustrate changes from an indication perspective [13] and for our primary indicators expressed antipsychotic use in the context of dementia as a percentage of people with dementia (defined as people with a prior anti-dementia medicine dispensing) and antipsychotic polypharmacy as a percentage of people receiving any antipsychotic.

\section{Patient factors associated with low-value prescribing practices}

For our primary indicators only, we stratified the rates of each low-value practice in 2016 by patient age, sex and comorbidity score. We categorised age at the time of dispensing as $65-74,75-84$ and $\geq 85$ years for benzodiazepines in the elderly and antipsychotics in the context of dementia and as 18-49, 50-64 and $\geq 65$ years for antipsychotic polypharmacy. We estimated the number of comorbidities for each individual in the population dispensed any medicine with the RxRisk score [14] using the 12 months of dispensings prior to the first dispensing of any medicine in 2016. For each practice, we excluded comorbidities from the RxRisk score that were part of the practice to avoid inflating associations and categorised the number of comorbidities as; $0-2,3-5,6-8$, and $\geq 9$.

To estimate the association of patient age, sex and comorbidity score with each low-value practice indicator in 2016, we ran crude and adjusted logistic regression analyses. We expressed associations as crude and adjusted odds ratios with 95\% confidence intervals, using males, the youngest age group and 0-2 comorbidities as reference categories.

\section{Direct medicine costs}

For our primary indicators only, we calculated direct medicine costs by identifying all dispensings associated with each low-value practice in 2016. For antipsychotic polypharmacy we summed the costs of all antipsychotics involved in a polypharmacy episode, as we were not able to determine appropriate monotherapy. We calculated medicine costs to the Australian government and patients based on co-payment thresholds and medicine cost at the date of dispensing using historical data supplied by the PBS Advisory Board. To obtain national cost estimates we multiplied these estimates from the PBS $10 \%$ sample by ten.

All analyses were performed with SAS, version 9.4 (SAS Institute Inc) and Stata version 12 (Statacorp).

\section{Results}

According to our indicators, we found little to no change in the annual rates of each low-value prescribing practice across the study years. The rates of both primary and secondary indicators are shown in Fig. 1 but henceforth we refer only to primary indicators. The rate of prevalent use of benzodiazepines in the elderly $(\geq 65$ year olds) was $16.5 \%$ in 2013 and $15.3 \%$ in 2016. Antipsychotic use in the context of dementia in the elderly was $0.4 \%$ in 2013 and $0.5 \%$ in 2016 and the rate of antipsychotic polypharmacy remained $0.2 \%$ throughout the study period.

Among people with dementia, 24.9\% received an antipsychotic in 2013 and 21.3\% in 2016. Among people who were dispensed an antipsychotic, 6.7\% received antipsychotic polypharmacy in 2013 and $7.1 \%$ in 2016 (Additional file 1: Figure S1a and b).

\section{Patient factors associated with low-value psychotropic prescribing}

In 2016, based on primary indicators; elderly women had greater adjusted odds than men of receiving benzodiazepines and the adjusted odds of receiving a benzodiazepine increased with patients' age and number of comorbidities (Fig. 2, Additional file 1: Table S2). The adjusted odds of receiving an antipsychotic in the context of dementia were independent of sex but increased with age and comorbidity. The adjusted odds of receiving antipsychotic polypharmacy decreased with age and increased with comorbidity (Fig. 2, Additional file 1: Tables S2, S3 and S4 for adjusted and crude odds ratios as well as stratified prevalence of each practice).

Based on the extrapolated costs of our primary indicators, the direct medicine cost of benzodiazepine use in the elderly in 2016 was $\$ 13.8$ million to patients and $\$ 12.2$ million to the government; for antipsychotic use in the context of dementia extrapolated costs were $\$ 0.7$ million to patients and $\$ 2.1$ million to the government; and for antipsychotic polypharmacy costs were $\$ 0.5$ million to patients $\$ 5.3$ million to the government (Table 2).

\section{Discussion}

Here we developed indicators within PBS dispensing claims to describe annual trends, associated patient factors 


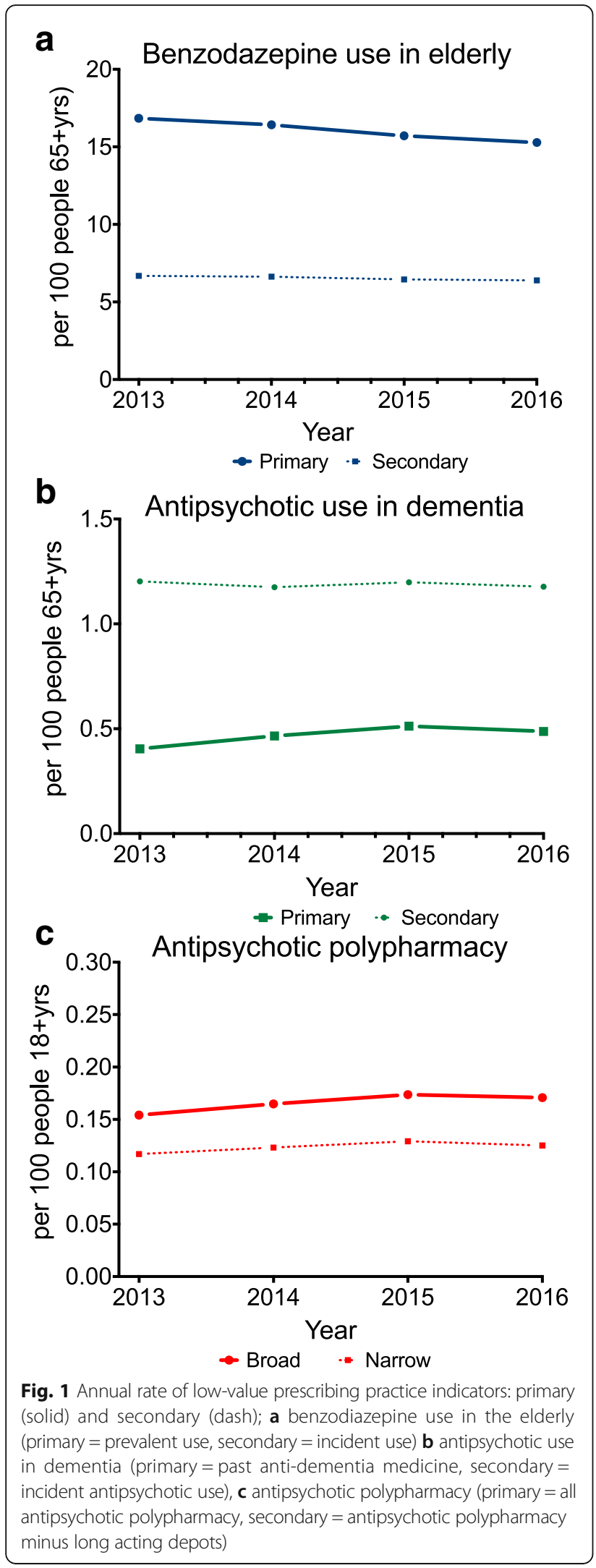

and direct medicine costs of three low-value prescribing practices as nominated by the Choosing Wisely campaign. According to these indicators, there were no major changes in the rate of any of these practices on a national level over the study period. This is despite long-standing evidence of the poor risk-benefit profiles of the practices described [15-17] and multiple national and local clinical interventions targeting these practices during this time [18]. There may however have been changes in the rates of these practices within smaller geographical areas that we did not examine.

While it may be too early to detect a measurable impact, according to our indicators, there has been no discernable change in the rates of these practices in relation to the launch of the Choosing Wisely Australia campaign in April 2015. However, only benzodiazepine use in the elderly and antipsychotic use in dementia were mentioned in the Choosing Wisely Australia campaign [19] and the wording of these practices specified indications and/or caveats. For instance the Australian and New Zealand Society of Geriatric Medicine states "do not prescribe benzodiazepines or other sedative-hypnotics to older adults as first choice for insomnia, agitation or delirium" and "do not use antipsychotics as the first choice to treat behavioral and psychological symptoms of dementia (BPSD)". Despite this it is likely that our measurement of benzodiazepine use in the elderly closely approximates the Australian variant of this practice, as benzodiazepines make up almost all sedative-hypnotic use in the elderly population. Furthermore, with the exceptions of epilepsy (clonazepam only) and anxiety, there would be few other indications that they would be used for in the elderly other than insomnia, agitation or delirium [20].

Caveats within these practices exemplify challenges inherent to measuring many Choosing Wisely recommendations in routinely collected data and suggest that quantification of these practices might not have been considered when lists of low-value practices were being constructed. For instance, it is difficult to determine whether antipsychotics are being used as 'first choice' in dementia when the preferred first choice is a non-pharmaceutical intervention that is not recorded in dispensing claims data. Similarly, don't routinely prescribe two or more antipsychotics concurrently also begs the question of how frequent 'routinely' actually is and on a clinical level this is open to inconsistent interpretation. Here we quantify prescribing practices defined as low-value a-priori by the Choosing Wisely campaign, and while there may be clinical circumstances in which such prescribing is appropriate we have taken these practices at face value. Historically, a number of other physician-led, consensus-based guidelines such as the Beers and STOPP criteria [21, 22] have been developed 


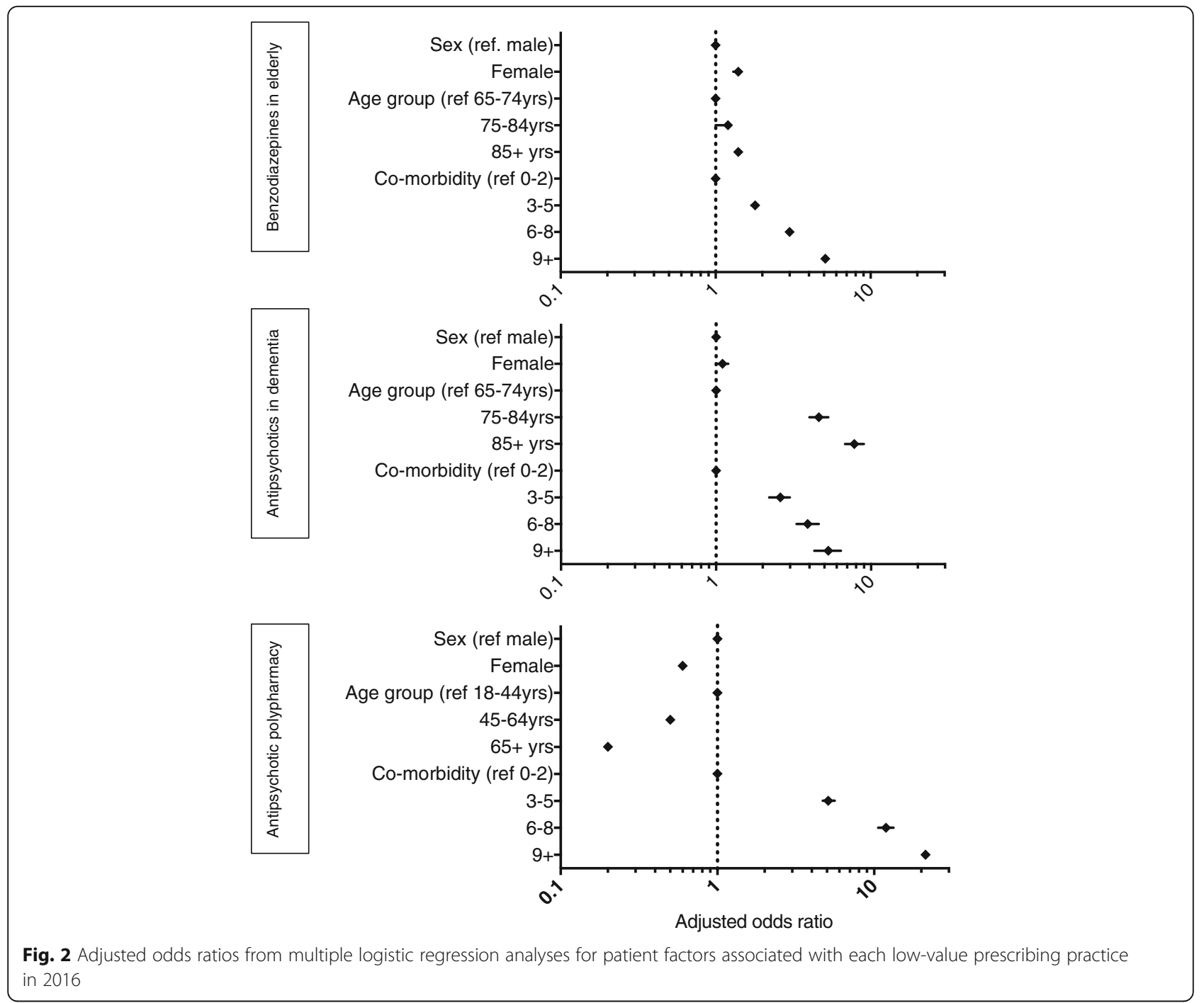

to identify potentially inappropriate prescribing practices and assist with de-prescribing, but these tend to focus on specific populations (such as the elderly).

Other measurement issues related to ascertainment become apparent when comparing this study to U.S. studies of antipsychotic use in dementia using Medicare data where dementia diagnoses from clinical encounter information are available. One such study showed mean annual rates of antipsychotic use in dementia to be higher (31\%) than our study $(21 \%$ in
2016), most likely because of more accurate dementia ascertainment [23]. Another UK study found that $15.2 \%$ of elderly patients with dementia had received an antipsychotic, but this study only included people prescribed 'low doses' within one primary care trust [24].

It is concerning, but not surprising, that the likelihood of receiving the first two practices increases with age, and the likelihood of receiving all three practices increases with comorbidity burden, as this is

Table 2 Number of dispensings and extrapolated direct medicine costs of three low-value prescribing practices in 2016

\begin{tabular}{lllll}
\hline & & \multicolumn{2}{l}{ Low-value prescribing practice } & \\
\cline { 3 - 5 } & & Benzodiazepines in elderly & Antipsychotics in dementia & Antipsychotic polypharmacy \\
\hline Number of dispensings (in PBS 10\% sample) & & 240,460 & 11,777 & 51,310 \\
Extrapolated direct medicine costs (/\$ million) & Patients & 13.8 & 0.7 & 0.5 \\
& Government & 12.2 & 2.1 & 5.3 \\
& Total & 26 & 2.8 & 5.8 \\
\hline
\end{tabular}


accompanied by a commensurate increase in the risk of adverse events associated with frailty, polypharmacy and drug-disease interactions [25]. Hence, caution is required when prescribing antipsychotics in this clinical context.

While the main focus of the Choosing Wisely campaign is to promote safe and effective care it is clear that low-value prescribing also incurs significant direct medication costs, totaling \$21 million AUD to the government in 2016. However, the majority of the cost of these practices is likely to be related to their clinical adverse effects [6]. For example, Table 3 illustrates how the cost to the government of excess hip fractures related to benzodiazepine use in the elderly may be in the region of \$29 million AUD annually. However, these potential cost savings do not account for the additional costs of alternative non-pharmacological treatments. This one adverse drug event, when added to direct medicine costs brings the total cost of benzodiazepine use in the elderly to $\$ 41$ million.

\section{Implications for policy and future research}

Our indicators of three low-value psychotropic prescribing practices have demonstrated a lack of any major changes in their frequency, suggesting a more concerted national effort is required to realize decreases in these practices and their related harms. Part of this process must be a deep understanding of the views of prescribers and the pressures they experience as well as synthesizing existing prescribing practice-specific intervention related literature [26]. The indicators developed here could be used to measure changes resulting from any ensuing interventions. Knowledge of patient characteristics associated with these practices also provides some focus for policy interventions. For instance, renewed efforts to target benzodiazepine and antipsychotic use in nursing homes is likely to have the highest yield as an initial

Table 3 Calculation of downstream costs of benzodiazepine use in the elderly related to hip fractures

Estimated adjusted rate ratio of hip fractures in elderly people ( $\geq 50$ years) taking benzodiazepines compared to those who were not $=1.52$ (95\% Cl: 1.37-1.68) [23]

Proportion of elderly people ( $\geq 65$ years) dispensed benzodiazepines (current study) $=0.067$

Estimated number of osteoporotic \& osteopenic hip fractures in $2016=26,232[34]$

Estimated average direct cost of a hip fracture in $2012=\$ 32,569$

AUD [34]

Proportion of hip fractures attributable to benzodiazepines

$=\frac{(1.52-1) * 0.067}{[(1.52-1) * 0.067)]+1}=0.033$

Number of hip fractures attributable to benzodiazepines $=0.033 * 26,232=883$

Excess costs of hip fractures related to benzodiazepine use in the elderly

$=883^{*} \$ 32,569=\$ 29$ million (95\% Cl: $\$ 20-\$ 38$ million) AUD approach as this population tends to be older and have more co-morbidity [27-29].

\section{Limitations}

Private prescribing is not captured within PBS dispensing claims and this is estimated to account for up to 10 and $6 \%$ of all benzodiazepine and antipsychotic prescribing respectively [30]. We did not include z-drugs in this study as they are not routinely captured in PBS data and they only account for a small proportion of sedative dispensings in Australia [30]. In this study we were unable to capture dose reductions amongst individuals taking these medicines in attempts by prescribers to minimise harm.

Medicines priced beneath the co-payment threshold were not captured within PBS data prior to July 2012 but this unlikely to have affected our results [8]. We made assumptions about the duration of exposure following one dispensing of a medicine in order to determine polypharmacy. Sensitivity analyses around these assumptions, published elsewhere [10] demonstrate that this is unlikely to affect trends but may underestimate the true prevalence of antipsychotic polypharmacy. The direct medicine costs of antipsychotic polypharmacy includes all antipsychotics involved in the polypharmacy episode as without clinical details we were unable to identify appropriate monotherapy. Therefore the cost of antipsychotic polypharmacy will be an overestimate. While the RxRisk score is predictive of mortality, it may not capture all co-morbidities [31]. Past dispensings claims for anti-dementia medicines only capture between 40 and $60 \%$ of people with dementia [32, 33], therefore our primary indicator of this practice will most likely under-estimate antipsychotic use in dementia. Applying the broader secondary indicator of people receiving new antipsychotic dispensings is likely to capture all people using antipsychotics in the context of dementia but may also capture antipsychotic use for other conditions such as delirium. We used a three-year look-back to identify past anti-dementia medicine dispensings, as we believe that this will capture most people with a prior dispensing of these medicines.

Finally, clinically contextual information such as the indication for which the medicine was prescribed and comorbidities was not available and hence we cannot be certain that prescribing was inappropriate. However, we have endeavored to quantify these three low-value practices as nominated by the Choosing Wisely campaign rather than to make judgments about the clinical appropriateness of prescribing in each instance.

\section{Conclusions}

The ultimate aim of campaigns such as Choosing Wisely is to optimize patient safety and quality of 
care. The lack of any substantial change in indicators of three low-value psychotropic prescribing practices described here, along with their relatively high rates and potential cost implications, indicates that renewed efforts are required to achieve further decreases in the rates of these practices.

\section{Additional file}

Additional file 1: Table S1. List of included medicines and ATC codes as well as list of item codes excluded to define secondary indicator for antipsychotic polypharmacy. Table S2. Low-value psychotropic prescribing practices, adjusted odds ratios of associated patient characteristics and direct medicine costs in 2016. Table S3. Crude odds ratios of associated patient characteristics in 2016 for each low-value psychotropic prescribing practice. Table S4. Prevalence of each low-value practice in 2016 based on primary indicators by sex and age group. Figure S1. Annual rate of low-value prescribing practice indicators; a) Antipsychotic use in dementia (defined by past anti-dementia medicine) b) Antipsychotic polypharmacy (all antipsychotics included). (DOCX $315 \mathrm{~kb}$ )

\section{Abbreviations}

ABS: Australian Bureau of Statistics; ATC: Anatomical Therapeutic Chemical Classification; AUD: Australian Dollars; BPSD: Behavioral and psychological symptoms of dementia; PBS: Pharmaceutical Benefits Scheme

\section{Acknowledgements}

We thank the Department of Human Services Australia for supplying the data. AGE receives salary support as the HCF Research Foundation Professorial Fellow, receives consulting/sitting fees from Cancer Australia, NPS MedicineWise (facilitator of Choosing Wisely Australia), the Australian Commission on Safety and Quality in Health Care (Atlas of Health Care Variation Advisory Group), the Queensland Government Department of Health, and the Australian Government Department of Health as an appointee to the MBS Review Taskforce.

\section{Funding}

This research was supported by the National Health and Medical Research Council (NHMRC) Centre for Research Excellence in Medicines and Ageing (CREMA), a Project Grant for measuring low-value care for targeted policy action and JB received funding support from an NHMRC Postgraduate award. These funding bodies played no role in the design of the study and collection, analysis, and interpretation of data or in writing the manuscript.

\section{Availability of data and materials}

The data that support the findings of this study are available from Department of Human Services of the Australian Commonwealth Government but restrictions apply to the availability of these data, which were used under license for the current study, and so are not publicly available. Data are however available from the authors upon reasonable request and with permission of the Department of Human Services of the Australian Commonwealth Government.

\section{Authors' contributions}

All authors made substantial contributions to conception and design of the study, SP and NB acquired the data, JB and BD analysed the data and all authors contributed data interpretation. All authors contributed to drafting and reviewing the final manuscript. All authors read and approved the final manuscript.

\section{Ethics approval and consent to participate}

The New South Wales Population and Health Services Ethics Committee (2013/11/494) approved relevant components of this study, including a waver of consent. Data access was granted by the Department of Human Services (Ml5250).

\section{Consent for publication}

A waiver of consent was granted for this project.

\section{Competing interests}

The authors declare that they have no competing interests.

\section{Publisher's Note}

Springer Nature remains neutral with regard to jurisdictional claims in published maps and institutional affiliations.

\section{Author details}

${ }^{1}$ Medicines Policy Research Unit, Centre for Big Data Research in Health, University of New South Wales, Sydney, NSW 2052, Australia. ${ }^{2}$ Centre of Public Health Sciences, Faculty of Medicine, University of Iceland, Reykjavik, Iceland. ${ }^{3}$ School of Medicine, The University of Sydney, Sydney, NSW, Australia. ${ }^{4}$ Menzies Centre for Health Policy, The University of Sydney, Sydney, Australia.

Received: 13 April 2018 Accepted: 11 December 2018 Published online: 29 December 2018

\section{References}

1. Scott I, Duckett S. In search of professional consensus in defining and reducing low-value care. Med J Aust. 2015;203(4):179-81.

2. Gliwa C, Pearson SD. Evidentiary rationales for the choosing wisely top 5 lists. J Am Med Assoc. 2014;311(14):1443-4.

3. Brody H. Medicine's ethical responsibility for health care reform-the top five list. N Engl J Med. 2010;362(4):283-5.

4. Levinson W, Kallewaard M, Bhatia RS, Wolfson D, Shortt S, Kerr EA, Burgers J, Cucic C, Daniels M, Forde I. 'Choosing wisely': a growing international campaign. BMJ Qual Saf. 2015;24:167-74.

5. Schwartz AL, Landon BE, Elshaug AG, Chernew ME, McWilliams JM. Measuring low-value care in Medicare. JAMA Intern Med. 2014;174(7): 1067-76.

6. Brett J, Elshaug AG, Bhatia RS, Chalmers K, Badgery-Parker T, Pearson S-A. A methodological protocol for selecting and quantifying low-value prescribing practices in routinely collected data: an Australian case study. Implement Sci. 2017;12(1):58.

7. Australian Atlas of Healthcare Variation. 2015. http://www.safetyandquality. gov.au/atlas/. [Accessed Jan 2018].

8. Mellish L, Karanges EA, Litchfield MJ, Schaffer AL, Blanch B, Daniels BJ, Segrave A, Pearson S-A. The Australian pharmaceutical benefits scheme data collection: a practical guide for researchers. BMC Res Notes. 2015:8(1):634.

9. ATC/DDD Index 2018. https://www.whocc.no/atc_ddd_index/. [Accessed Jan 2018].

10. Brett J, Daniels B, Karanges EA, Buckley NA, Schneider C, Nassir A, McLachlan AJ, Pearson SA. Psychotropic polypharmacy in Australia, 2006 to 2015: a descriptive cohort study. Br J Clin Pharmacol. 2017;83(11):2581-8.

11. Leckman-Westin E, Kealey E, Gupta N, Chen Q, Gerhard T, Crystal S, Olfson M, Finnerty M. Validation of a claims-based antipsychotic polypharmacy measure. Pharmacoepidemiol Drug Saf. 2014;23(6):628-35.

12. ABS.STAT. http://stat.data.abs.gov.au/Index.aspx?DataSetCode=ERP_ QUARTERLY. Accessed Jan 2018.

13. Chalmers K, Pearson S-A, Elshaug AG. Quantifying low-value care: a patientcentric versus service-centric lens. BMJ Qual Saf. 2017;26:855-8.

14. Vitry A, Wong SA, Roughead EE, Ramsay E, Barratt J. Validity of medicationbased co-morbidity indices in the Australian elderly population. Aust N Z J Public Health. 2009;33(2):126-30

15. Lader M. Benzodiazepines revisited —will we ever learn? Addiction. 2011; 106(12):2086-109.

16. Gareri P, De Fazio P, De Fazio S, Marigliano N, Ibbadu GF, De Sarro G. Adverse effects of atypical antipsychotics in the elderly. Drugs Aging. 2006; 23(12):937-56

17. Gallego JA, Nielsen J, De Hert M, Kane JM, Correll CU. Safety and tolerability of antipsychotic polypharmacy. Expert Opin Drug Saf. 2012;11(4):527-42.

18. National Prescribing Service MedicineWise. 2018. https://www.nps.org.au/. [Accessed Jan 2018].

19. Choosing Wisely Australia. 2018. http://www.choosingwisely.org.au/. [Accessed Jan 2018].

20. Maust DT, Kales HC, Wiechers IR, Blow FC, Olfson M. No end in sight: benzodiazepine use in older adults in the United States. J Am Geriatr Soc. 2016;64(12):2546-53 
21. Campanelli CM. American Geriatrics Society updated Beers Criteria for potentially inappropriate medication use in older adults: the American Geriatrics Society 2012 Beers Criteria Update Expert Panel. J Am Geriatr Soc. 2012:60(4):616.

22. Gallagher P, Ryan C, Byrne S, Kennedy J, O'Mahony D. STOPP (Screening Tool of Older Person's Prescriptions) and START (Screening Tool to Alert doctors to Right Treatment). Consensus validation. Int I Clin Pharmacol Ther. 2008;46(2):72-83.

23. Colla CH, Morden NE, Sequist TD, Schpero WL, Rosenthal MB. Choosing wisely: prevalence and correlates of low-value health care services in the United States. J Gen Intern Med. 2015;30(2):221-8.

24. Child A, Clarke A, Fox C, Maidment I. A pharmacy led program to review anti-psychotic prescribing for people with dementia. BMC Psychiatry. 2012; 12(1):155

25. Routledge PA, O'Mahony M, Woodhouse K. Adverse drug reactions in elderly patients. Br J Clin Pharmacol. 2004;57(2):121-6.

26. Cabana MD, Rand CS, Powe NR, Wu AW, Wilson MH, Abboud P-AC, Rubin HR. Why don't physicians follow clinical practice guidelines?: a framework for improvement. J Am Med Assoc. 1999;282(15):1458-65.

27. Hilmer SN, Gnjidic D. Rethinking psychotropics in nursing homes. Med J Aust. 2013;198(2):77.

28. Maidment ID, Shaw RL, Killick K, Damery S, Hilton A, Wilcock J, et al. Improving the management of behaviour that challenges associated with dementia in care homes: protocol for pharmacy-health psychology intervention feasibility study. BMJ Open. 2016;6(3):e010279.

29. Ailabouni N, Mangin D, Nishtala PS. Deprescribing anticholinergic and sedative medicines: protocol for a feasibility trial (DEFEAT-polypharmacy) in residential aged care facilities. BMJ Open. 2017;7(4):e013800.

30. Hollingworth SA, Siskind DJ. Anxiolytic, hypnotic and sedative medication use in Australia. Pharmacoepidemiol Drug Saf. 2010;19(3):280-8.

31. Lu CY, Barratt J, Vitry A, Roughead E. Charlson and Rx-risk comorbidity indices were predictive of mortality in the Australian health care setting. J Clin Epidemiol. 2011;64(2):223-8.

32. Goeman D, Harvey K, Lee CY, Petrie N, Beanland C, Culhane C, Koch S. How prolific is psychotropic medicines use in people with dementia in Australia within the community setting? A retrospective analysis. Drugs Real World Outcomes. 2015:2(3):289-98.

33. Rattinger GB, Burcu M, Dutcher SK, Chhabra PT, Rosenberg PB, SimoniWastila L, Franey CS, Walker LD, Zuckerman IH. Pharmacotherapeutic management of dementia across settings of care. J Am Geriatr Soc. 2013; 61(5):723-33.

34. Watts JJ, Abimanyi-Ochom J, Sanders KM. Osteoporosis costing all Australian: a new burden of disease analysis-2012 to 2022; 2013.

Ready to submit your research? Choose BMC and benefit from:

- fast, convenient online submission

- thorough peer review by experienced researchers in your field

- rapid publication on acceptance

- support for research data, including large and complex data types

- gold Open Access which fosters wider collaboration and increased citations

- maximum visibility for your research: over $100 \mathrm{M}$ website views per year

At $\mathrm{BMC}$, research is always in progress.

Learn more biomedcentral.com/submissions 